ARTICLE

Received 6 Feb 2015 | Accepted 19 Aug 2015 | Published 25 Sep 2015

DOI: $10.1038 /$ ncomms 9401

OPEN

\title{
Light-induced pyroelectric effect as an effective approach for ultrafast ultraviolet nanosensing
}

\author{
Zhaona Wang ${ }^{1,2, \star}$, Ruomeng $\mathrm{Yu}^{1, \star}$, Caofeng Pan ${ }^{3}$, Zhaoling $\mathrm{Li}^{1}$, Jin Yang ${ }^{1}$, Fang $\mathrm{Yi}^{1} \&$ Zhong Lin Wang ${ }^{1,3}$
}

Zinc oxide is potentially a useful material for ultraviolet detectors; however, a relatively long response time hinders practical implementation. Here by designing and fabricating a self-powered $\mathrm{ZnO}$ /perovskite-heterostructured ultraviolet photodetector, the pyroelectric effect, induced in wurtzite $\mathrm{ZnO}$ nanowires on ultraviolet illumination, has been utilized as an effective approach for high-performance photon sensing. The response time is improved from $5.4 \mathrm{~s}$ to $53 \mu \mathrm{s}$ at the rising edge, and $8.9 \mathrm{~s}$ to $63 \mu \mathrm{s}$ at the falling edge, with an enhancement of five orders in magnitudes. The specific detectivity and the responsivity are both enhanced by $322 \%$. This work provides a novel design to achieve ultrafast ultraviolet sensing at room temperature via light-self-induced pyroelectric effect. The newly designed ultrafast self-powered ultraviolet nanosensors may find promising applications in ultrafast optics, nonlinear optics, optothermal detections, computational memories and biocompatible optoelectronic probes.

\footnotetext{
${ }^{1}$ School of Materials Science and Engineering, Georgia Institute of Technology, Atlanta, Georgia 30332-0245, USA. ${ }^{2}$ Department of Physics, Applied Optics Beijing Area Major Laboratory, Beijing Normal University, Beijing 100875, China. ${ }^{3}$ Beijing Institute of Nanoenergy and Nanosystems, Chinese Academy of Sciences, Beijing 100083, China. * These authors contributed equally to this work. Correspondence and requests for materials should be addressed to Z.L.W. (email: zhong.wang@mse.gatech.edu).
} 
s one of the most widely used wurtzite semiconductor materials, $\mathrm{ZnO}$ has been extensively investigated as building blocks for nano/microdevices in various applications, including energy harvester ${ }^{1}$, optoelectronics ${ }^{2,3}$, field-effect transistors ${ }^{4}$ and logic computations ${ }^{5,6}$. Among these applications, room temperature ultraviolet detection is drawing tremendous attentions for defence technology, flame warning and chemical/biological analysis ${ }^{7,8}$ because of the wide bandgap $(3.3 \mathrm{eV})$ and high exciton-binding energy $(60 \mathrm{meV})$ of $\mathrm{ZnO}$ (refs 9-12). Although numerous $\mathrm{ZnO}$-based ultraviolet photodetectors (PDs) have been demonstrated with high photoresponsivity and/or specific detectivity, the relatively long response time and fluctuations in performance have always been an inevitable obstacle to improving the general performances of these PDs for practical applications. For example, the typical rising and falling times of previously reported $\mathrm{ZnO}$-based ultraviolet PDs are ranging from a few seconds to few hours in most cases ${ }^{13-17}$. Therefore, it is necessary and essential to develop ultrafast response ultraviolet sensing devices based on $\mathrm{ZnO}$ $\mathrm{micro} /$ nanostructures.

Owing to the non-central symmetric crystal structures of wurtzite $\mathrm{ZnO}$, pyroelectric potentials are induced by temperature changes across the material ${ }^{18-20}$. On light illuminations, a rapid rise in temperature is naturally induced within $\mathrm{ZnO}$ nanowires (NWs), leading to a distribution of pyroelectric potentials along the crystal with polarization pyrocharges presenting at both ends of the NW. A new approach is thus introduced to modify the charge-carrier transport process during the optoelectronic processes within $\mathrm{ZnO}$-based devices by utilizing the pyroelectric polarizations.

In this work, we utilize this light-self-induced pyroelectric effect in $\mathrm{ZnO}$ to modulate the optoelectronic processes and thus enhance the performances of ultraviolet sensing. To maximize the enhancements of pyroelectric effect, low background temperature is maintained by minimizing the dark current $(<0.1 \mathrm{nA})$ via designing and fabricating a self-powered PD that operates under zero bias $8,21,22$. Considering the simple synthesizing process and intrinsic optoelectronic properties, such as direct bandgap, large absorption coefficient and high carrier mobility ${ }^{23-29}$, $\mathrm{CH}_{3} \mathrm{NH}_{3} \mathrm{PbI}_{3} \quad\left(\mathrm{MAPbI}_{3}\right)$ perovskite is applied to form a heterostructure with $\mathrm{ZnO}$ as self-powered PDs. The response time of the self-powered $\mathrm{ZnO}$ /perovskite-heterostructured $(\mathrm{ZPH})$ $\mathrm{PD}$ is improved from $5.4 \mathrm{~s}$ to $53 \mu \mathrm{s}$ in the rising time, and $8.9 \mathrm{~s}$ to $63 \mu \mathrm{s}$ in the falling time by five orders in magnitudes. The specific detectivity $D^{*}$ and the responsivity $R$ of the PD are both enhanced by $322 \%$. The light-self-induced pyroelectric effect is attributed to these enhancements, and the working mechanism is carefully investigated. This work provides a novel design approach to achieve ultrafast response ultraviolet-sensing devices at room temperature enhanced by pyroelectric effect. The demonstrated self-powered PDs may become prospective candidates for ultrafast and energy-efficient ultraviolet sensing in different research and commercial fields.

\section{Results}

Design mechanism of self-powered ZPH PDs. The structure of self-powered ZPH PD is schematically illustrated in Fig. 1a. Side-view and top-view scanning electron microscopy images presented in Fig. $1 \mathrm{~b}$ confirm the PD configuration, showing fluorine-doped tin oxide (FTO) glass uniformly and compactly covered by $\mathrm{ZnO}-\mathrm{NW}$ array with diameters of $50-70 \mathrm{~nm}$ and lengths of $500 \mathrm{~nm}$ (top right panel of Fig. 1b, Supplementary Fig. 1a and Supplementary Note 1), and then partially covered by $\mathrm{MAPbI}_{3}$ perovskite with an average thickness of $500 \mathrm{~nm}$ (bottom right panel of Fig. 1b, Supplementary Fig. 1b and Supplementary
Note 1), followed by a complete cover of hole transport material (HTM) spiro-OMeTAD with thickness of $500-1,000 \mathrm{~nm}$ (Supplementary Fig. 1c and Supplementary Note 1) and $\mathrm{Cu}$ top electrode with a thickness of $250 \mathrm{~nm}$. The phase of $\mathrm{MAPbI}_{3}$ perovskite is verified by taking XRD spectra (Fig. 1c) of the perovskite film-covered $\mathrm{ZnO}-\mathrm{NW}$ array ${ }^{30}$. Detailed fabrication processes and measurement set-up are found in the Methods.

Relevant energy levels of the materials used to fabricate the self-powered ZPH PDs are depicted in Fig. 1d. The valence band and conduction band of the $\mathrm{MAPbI}_{3}$ perovskite are -5.4 and $-3.9 \mathrm{eV}$, versus vacuum, respectively ${ }^{25,31}$. On ultraviolet illumination, excitons are generated in the $\mathrm{ZnO}$ layer according to the absorption spectra of $\mathrm{ZnO}-\mathrm{NW}$ array and $\mathrm{ZnO} / \mathrm{MAPbI}_{3}$ perovskite film as shown in Supplementary Fig. $1 \mathrm{~d}$ and in Supplementary Note 1. The energy band alignment of the device ensures electrons are injected from the photoexcited layer of the $\mathrm{ZnO}$ and collected by FTO electrode while the valence band holes reach the $\mathrm{Cu}$ electrode through the hole transport layer.

Ultraviolet illumination produces a fast temperature increase within pyroelectric $\mathrm{ZnO} \mathrm{NW}$ s for the light absorption, due to the non-central symmetric crystal structures; pyroelectric potentials are induced across the NWs by polarizations, with positive potentials at the $\mathrm{HTM} / \mathrm{Cu}$ electrode side and negative potentials at the FTO electrode side based on the $c$ axis direction of ZnO-NW array, as shown in Fig. 1e. This light-self-induced pyroelectric potentials are aligning with the photovoltaic direction over the external circuit and thus enhance the transient short-circuit current and open-circuit voltage. More importantly, the pyroelectric potential presenting in $\mathrm{ZnO}$ NWs may speed up charge-carrier separation at the interface, preventing photoexcited electrons in $\mathrm{ZnO}$ from recombining with holes at the heterojunctions, further improving the performance of ultraviolet $\mathrm{PD}$ in response time. $I-V$ characteristics of the self-powered $\mathrm{ZPH}$ PDs under 325-nm illumination (red curve in Fig. 1f) were measured and plotted in Fig. If in comparison with dark current (black curve in Fig. 1f), showing a good ultraviolet response of the device in output current, increasing from $1.5 \mu \mathrm{A}$ (dark) to $143 \mu \mathrm{A}$ $\left(3.7 \times 10^{-3} \mathrm{~W} \mathrm{~cm}^{-2}\right)$ when the HTM/Cu electrode was biased at $0.5 \mathrm{~V}$. The self-powered operations are observed from the enlarged $I-V$ curves (right panel of Fig. 1f) with a measurable $(\sim 1 \mu \mathrm{A})$ photocurrent under ultraviolet illumination at zero bias. This can be attributed to the well-aligned band structure of the device (see Fig. 1d).

General performances of the self-powered ZPH PDs. The shortcircuit current response of ZPH PDs to ultraviolet illumination was systematically investigated and summarized in Fig. 2a by varying the power densities from $3.7 \times 10^{-3}$ to $9 \times 10^{-6}$ $\mathrm{W} \mathrm{cm}-2$. Under each power density, obvious two-stage shortcircuit currents were derived. In the first stage, a sharp peak was induced by the combination of photovoltaic effect and pyroelectric effect on illumination which lead to an instantaneous temperature rise within $\mathrm{ZnO}$. The corresponding short-circuit current is labelled as $I_{\text {pyro + photo; }}$ subscripts 'pyro' and 'photo' indicate the output current induced by pyroelectric effect and photovoltaic effect, respectively. In the second stage, the pyroelectric potentials disappeared as the temperature stayed constant by retaining the ultraviolet illumination, and the short-circuit current reached a steady plateau until shutting the laser beam, labelled as $I_{\text {photo }}$. The corresponding currents of both stages were extracted and plotted in Fig. $2 b$ as black and red dots, respectively, to demonstrate the pyroelectric enhancements on output signals of ZPH PDs. It is clear that the short-circuit currents $I_{\text {pyro + photo }}$ and $I_{\text {photo }}$ monotonically increase with the power density of illumination in both 

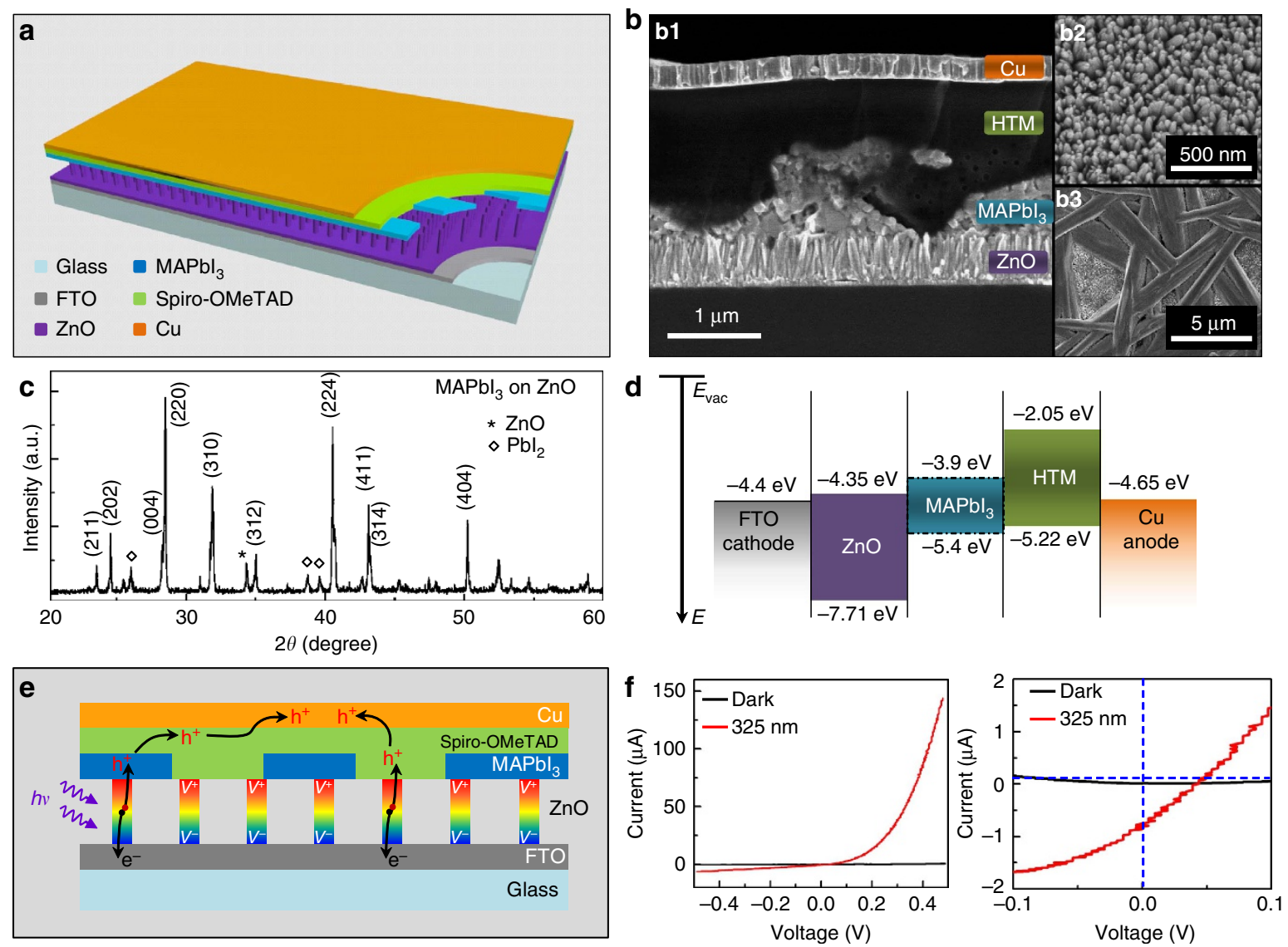

Figure 1 | Structure, characterization and design mechanism of self-powered ZPH PDs. (a) Schematic demonstration of the structure of self-powered ZPH PDs. FTO acts as the transparent electrode. (b) Scanning electron microscopy images of self-powered ZPH PDs: (b1) side view of ZnO coated by perovskite, hole transport material (Spiro-OMeTAD) and Cu electrode in sequence (b2,b3) top view of ZnO NWs array (b2) before and (b3) after being spin-coated by perovskite. (c) XRD spectra of the perovskite $\left(\mathrm{MAPbl}_{3}\right.$ ) on the $\mathrm{ZnO} \mathrm{NWs}$ layer. (d) Energy band diagram of a self-powered ZPH PD. Energies are expressed in electron volts, using the electron energy in vacuum as a reference. The energy levels of the conduction band edges of $\mathrm{ZnO}$, $\mathrm{MAPbl}_{3}$ and spiro-OMeTAD are at $-4.35,-3.9$ and $-2.05 \mathrm{eV}$, respectively, and the valence band edge of the perovskite is at $-5.4 \mathrm{eV}$. (e) Schematic illustration of the working mechanism of self-powered ZPH PDs. (f) I-V characteristics of the self-powered ZPH PDs under dark and 325-nm laser illumination with a power density of $3.7 \mathrm{~mW} \mathrm{~cm}^{-2}$

stages, with the current of the first stage larger than that of the second stage for all illumination conditions.

To better understand the enhancements by pyroelectric effect on the performances of ZPH PDs, specific detectivity $D^{\star}$ is demonstrated in Fig. 2c for currents obtained from both stages, defined as $D^{*}=R /\left(2 e \cdot I_{\text {dark }} / S\right)^{0.5}$ to describe the smallest detectable signal by considering the dark current $I_{\text {dark }}$, which is in the order of $10^{-11} \mathrm{~A}$ (Supplementary Fig. 3), as the major noise ${ }^{32} . S$ is the effective area of the PDs, $R$ is the corresponding photoresponsivity (Supplementary Fig. 2) calculated as $R=\frac{I_{\text {light }}-I_{\text {dark }}}{P_{\text {ill }}}=\frac{\eta_{\text {ext }} q}{h v} \cdot \gamma_{\mathrm{G}}$ (ref. 33), where $P_{\text {ill }}=I_{\text {ill }} \times S$ is the illumination power on PDs; $I_{\text {light }}$ represent the short-circuit current with illumination, respectively; $\gamma_{\mathrm{G}}$ is the internal gain; $\eta_{\text {ext }}$ is the external quantum efficiency; $q$ is electronic charge; $h$ is Planck's constant; $v$ is the frequency of the light; and $I_{\text {ill }}$ is the excitation power density. Both $D^{*}$ and $R$ derived from the first stage with a combination of pyroelectric effect and photovoltaic effect (labelled as $D_{\text {pyro + photo }}^{*}$ display larger values than those obtained from the second stage, where only photo-excitation process contributes (labelled as $D_{\text {photo }}^{*}$ ). The maximum value of $D^{*}$ is observed as $4.0 \times 10^{10}$ and $1.3 \times 10^{10}$ Jones for the first and second stages at the power density of $1.9 \times 10^{-5} \mathrm{~W} \mathrm{~cm}^{-2}$, respectively. Under the same condition, the maximum value of $R$ is 26.7 and $8.3 \mathrm{~mA} \mathrm{~W}^{-1}$ for two stages (Supplementary Note 2).
These results indicate $332 \%$ enhancements on both detectivity and photoresponsivity by pyroelectric effect.

The stability and repeatability of the self-powered ZPH PDs are studied and presented in Supplementary Figs 4 and 5 and in Supplementary Note 3 . Short-circuit $I-t$ curves of a typical device were measured under a power density of $3.7 \times 10^{-3} \mathrm{~W} \mathrm{~cm}^{-2}$ after keeping the device in air for 1 and 3 weeks (Supplementary Fig. 4), showing impressive stability of these PDs. Besides, the repeatability of the $\mathrm{PD}$ was also investigated by monitoring the $I-t$ performances of four different devices fabricated through the same procedures as shown in Supplementary Fig. 5. Considering the self-powered operation mechanism of $\mathrm{ZPH}$ PDs, open-circuit voltage, similar to short-circuit current indicated above, can also be utilized to detect the illumination intensities as shown in Supplementary Fig. 6. The open-circuit $V-t$ curves present a sharp peak followed by a stable plateau as well, with the open-circuit voltage in both stages increased monotonously with the power density (Supplementary Note 4).

Working mechanism of the self-powered ZPH PDs. Shortcircuit $I-t$ characteristics of the self-powered ZPH PDs were measured under $325 \mathrm{~nm}$ (top panel, Fig. 3a) and $442 \mathrm{~nm}$ (bottom panel, Fig. 3a) illuminations through an optical chopper with a time ratio of $1: 1$ at $100 \mathrm{~Hz}$ to investigate the physical mechanism 
a
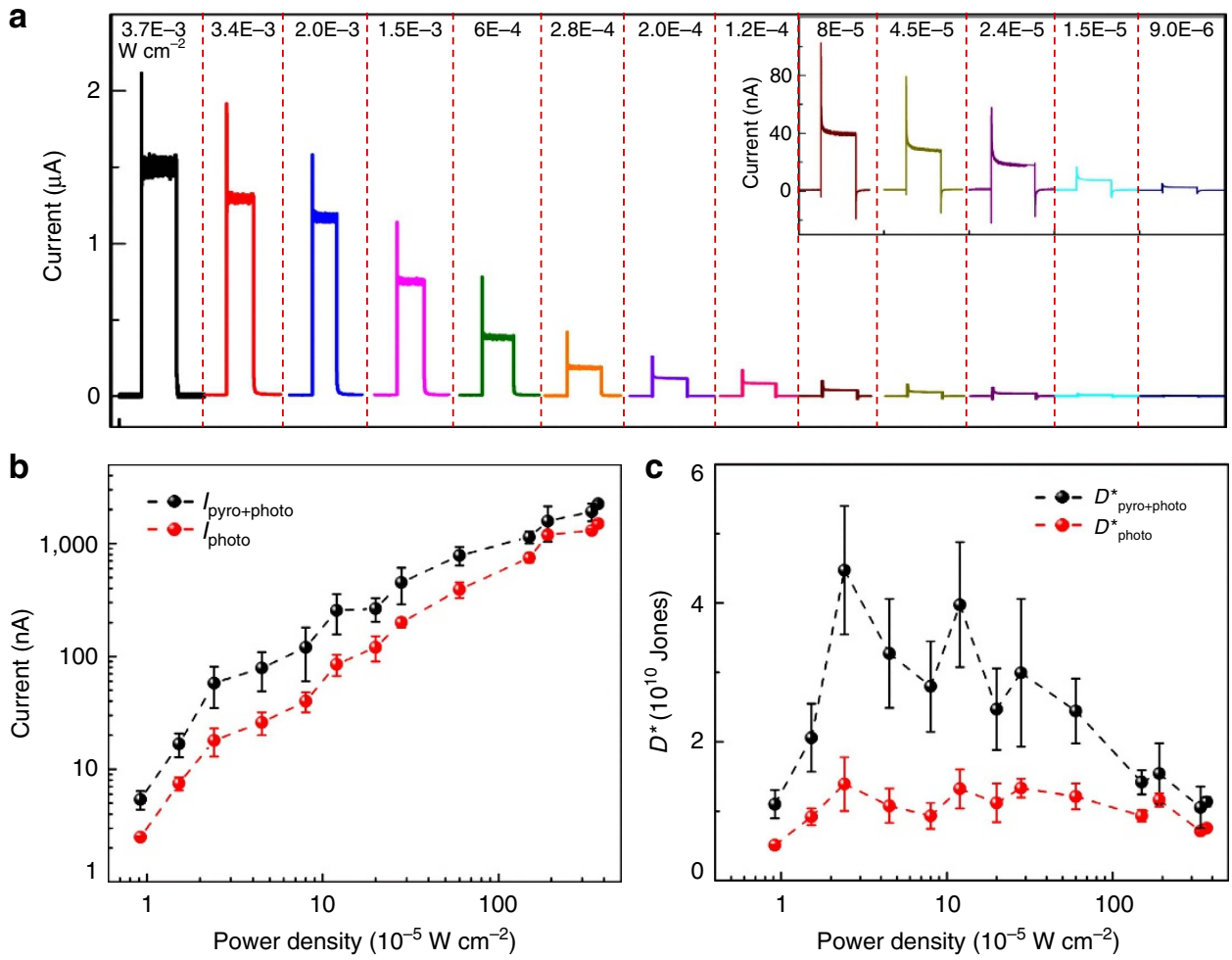

Figure 2 | Pyroelectric effect enhanced performances of self-powered ZPH PDs. (a) I-t characteristics of self-powered ZPH PDs under 325-nm illuminations with different power densities from $3.7 \times 10^{-3}$ to $9.0 \times 10^{-6} \mathrm{~W} \mathrm{~cm}^{-2}$, the inset is the enlarged $l-t$ curves under the corresponding illumination conditions. (b) The short-circuit current response (c) and specific detectivity $D^{\star}$ response of pyroelectric effect-combined photoexcitation process (black dots) and photoexcitation process (red dots), showing the enhancements by pyroelectric effect. Data reported in $\mathbf{b}, \mathbf{c}$ were calculated from l-t curves acquired over 20 times within 30 days under different power densities.
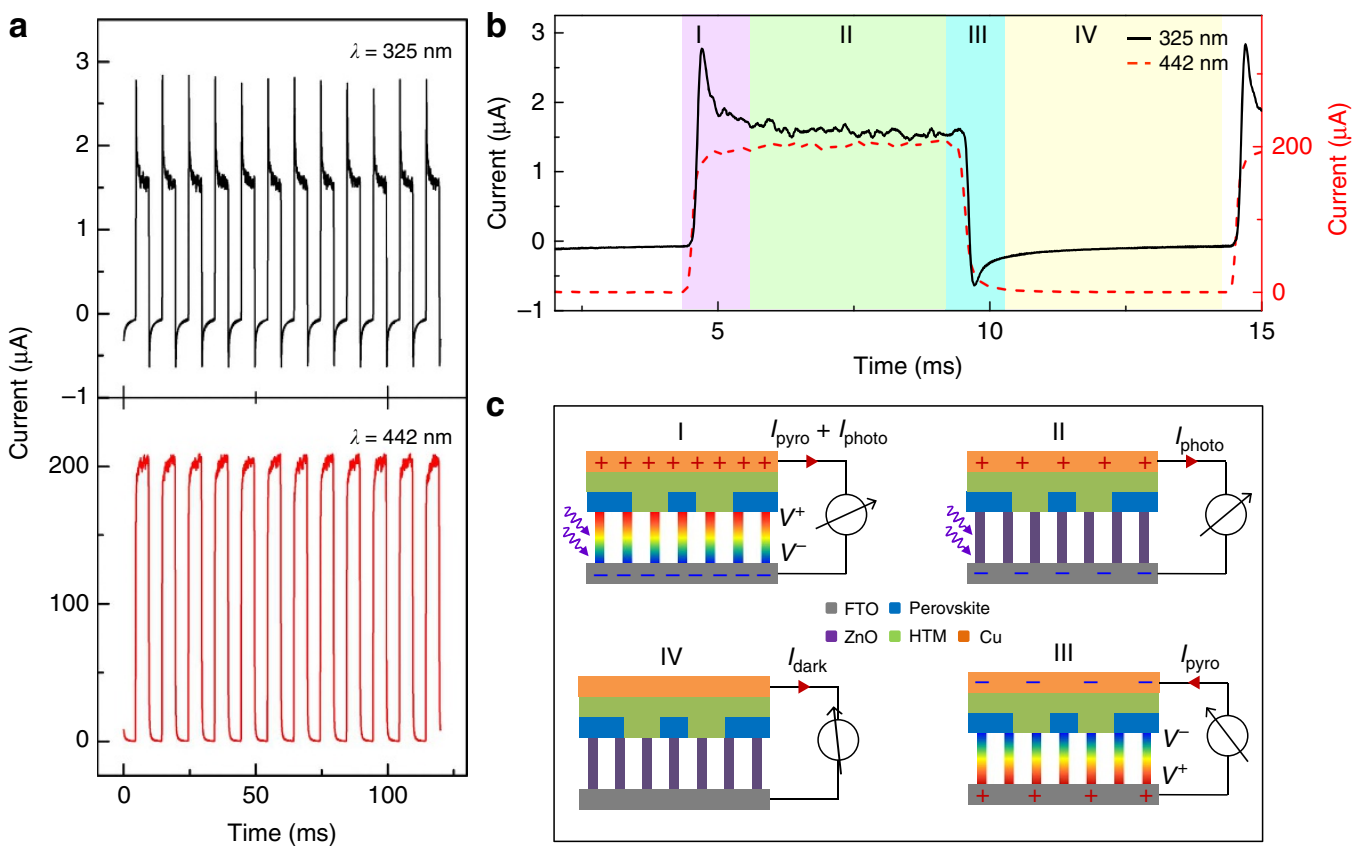

C

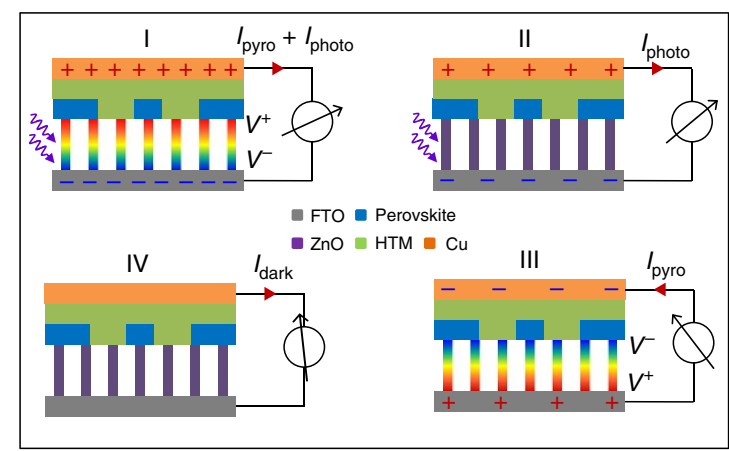

Figure 3 | Working mechanism of self-powered ZPH PDs. (a) I-t characteristics of the self-powered ZPH PDs under $325 \mathrm{~nm}$ (top) and $442 \mathrm{~nm}$ (bottom) laser illuminations through an optical chopper with a time ratio of 1:1 at $100 \mathrm{~Hz}$. (b) Enlarged plot of a single output period as shown in a for both $325-$ and 442-nm illuminations, divided into four stages, labelled as ' $I$ ', 'II', 'III' and 'IV'. (c) Schematic illustration of the working mechanism of pyroelectric effect-combined photoexcitation processes, corresponding to the four stages labelled in $\mathbf{b}$.

of photon-sensing process combined with pyroelectric effect. On the basis of the absorbance spectra presented in Supplementary Fig. 1d, ZnO NWs are transparent to 442-nm illumination, and therefore the pyroelectric effect was only observed under $325 \mathrm{~nm}$ as clearly shown in Fig. 3a,b. The signal of 325-nm illumination displays a sharp rising edge, a stable plateau 

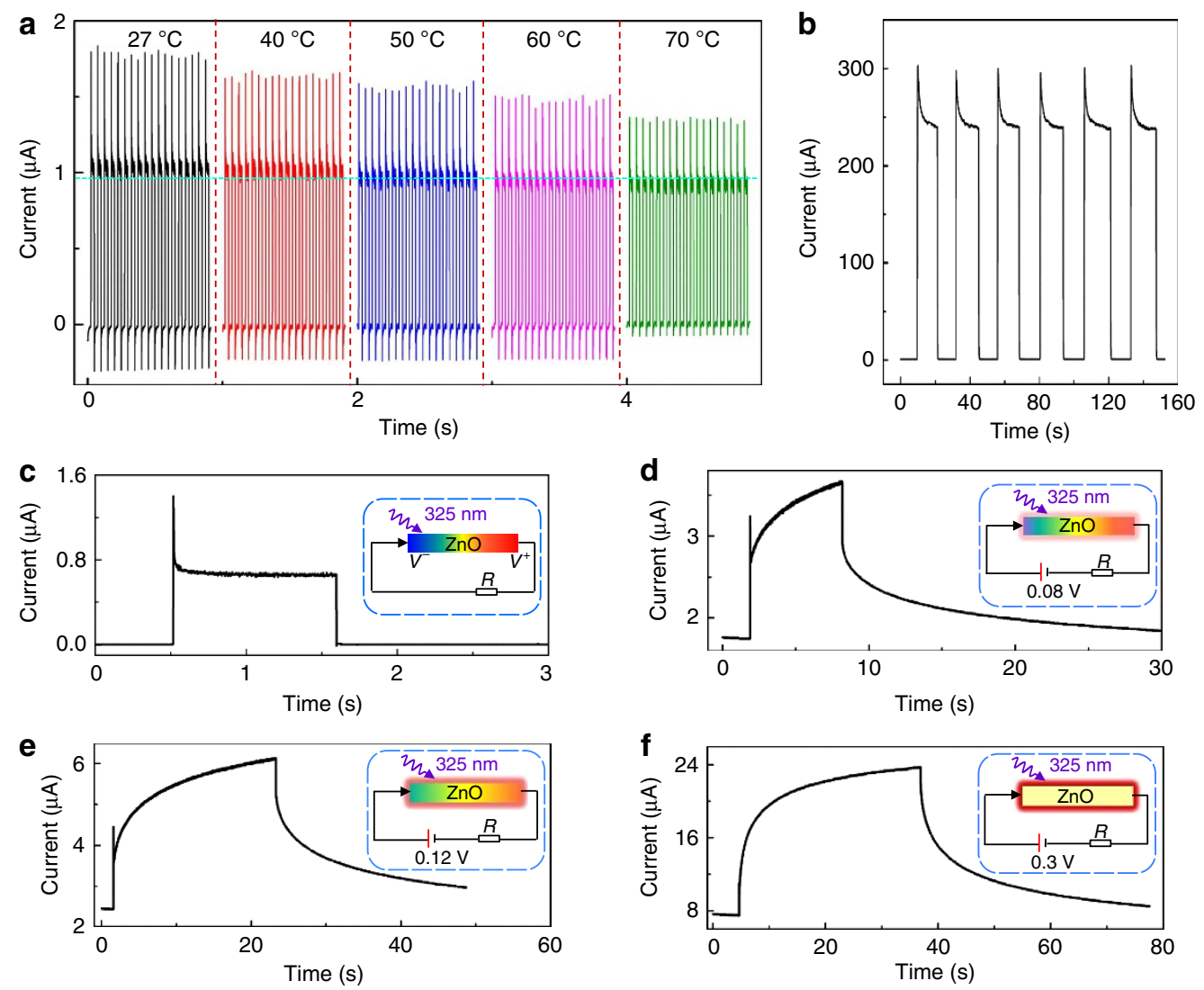

Figure 4 | Pyroelectric effect enhancements on response time of self-powered ZPH PDs. (a) I- $t$ response of the self-powered ZPH PDs to 325-nm illumination (power density of $1.8 \mathrm{~mW} \mathrm{~cm}^{-2}$ ) measured under different background environmental temperatures to confirm the pyroelectric effect. (b) I- $t$ curves of the $\mathrm{p}-\mathrm{Si} / \mathrm{ZnO} \mathrm{PD}$ biased at $-2.0 \mathrm{~V}$ under $325-\mathrm{nm}$ laser illumination with a power density of $2.6 \times 10^{-4} \mathrm{Wcm}^{-2}$. (c-f) $1-t$ curves of the ZPH PDs under different bias voltages of (c) $0.0 \mathrm{~V},(\mathbf{d}) 0.08 \mathrm{~V},(\mathbf{e}) 0.12 \mathrm{~V}$ and (f) $0.3 \mathrm{~V}$ to calculate the improved response time by pyroelectric effect. The inset indicates the corresponding circuit diagram with current heating effect (red glow around $\mathrm{ZnO}$ ) and pyroelectric effect of $\mathrm{ZnO}$ (colour code of $\mathrm{ZnO}$ ).

and a falling edge (black solid curve in Fig. 3b), while the signal of 442-nm illumination (red dash curve in Fig. $3 \mathrm{~b}$ ) is the same as those derived from traditional $\mathrm{ZnO}$-based PDs reported previously ${ }^{13-16}$. Additional measurements were conducted to investigate the frequency dependence of self-powered ZPH PDs under both 325- and 442-nm illuminations. The corresponding I- $t$ curves are presented in Supplementary Fig. 7, derived by varying the optical chopper frequency from 100 to $1,000 \mathrm{~Hz}$. No obvious dependence on frequency was observed under $325-\mathrm{nm}$ illumination, suggesting a potential ultrafast response of PDs enhanced by pyroelectric effect. As a comparison, the output signals were clearly affected by the frequency of light source under 442-nm illumination, indicating a slow response of these PDs without pyroelectric enhancements (Supplementary Note 5).

One cycle of the short-circuit $I-t$ curve under $325 \mathrm{~nm}$ was divided into four stages, labelled as I, II, III and IV in Fig. 3b,c, to elaborate the physical mechanism of the pyroelectric effectcombined photon-sensing performances of self-powered $\mathrm{ZPH}$ PDs. In stage 'I', the $325-\mathrm{nm}$ ultraviolet illumination generates free carriers as photocurrent to transport through the external circuits from $\mathrm{Cu}$ to FTO electrode; meanwhile, an instantaneous temperature increase inside $\mathrm{ZnO}$ is produced on ultraviolet illumination, leading to a distribution of polarization pyroelectric charges across the NW with positive pyro-potential at the $\mathrm{HTM} / \mathrm{Cu}$ electrode side, and negative pyro-potential at the FTO electrode side. The light-self-induced pyro-potential follows the photocurrent direction in external circuits and thus enhances the output signals with a sharp rising edge (I, Fig. 3 b,c). In stage 'II', the illumination is retained and the temperature stays at constant.
Therefore, the pyroelectric potential disappears rapidly (in less than $1 \mathrm{~ms}$ ) due to the leakage, and the output current reaches a stable plateau (II, Fig. 3b,c). In stage 'III', the illumination is eliminated and the photocurrent thus disappears, while an instantaneous temperature decrease induced during this process results in a pyro-potential distribution in the opposite direction to that in 'I', leading to current flows from FTO to $\mathrm{Cu}$ electrode as a falling peak with negative current magnitudes (III, Fig. 3b,c). In stage 'IV', the temperature falls back to room temperature and stays steady; thus, the pyro-potential vanishes again because of leakage and the output returns to dark current (IV, Fig. 3b,c).

The heating process of ultraviolet illumination was simulated by using finite element method based on the transient heat conduction equation as demonstrated in Supplementary Note 6. The temperature profiles, temperature variation rate $\mathrm{d} T / \mathrm{d} t$ of the $\mathrm{ZnO} \mathrm{NW}$ and the corresponding output currents induced by the pyroelectric effect were calculated and presented in Supplementary Fig. 8a,b. Furthermore, a nanosecond-pulsed laser with wavelength of $355 \mathrm{~nm}$ was applied to experimentally verify the pyroelectric response of $\mathrm{ZPH} \mathrm{PD}$ as shown in Supplementary Fig. 8c,d. These theoretical simulations and experimental results provide convinced proofs to establish the direct correlation between observed optoelectronic performances and the ultraviolet illumination-induced pyroelectric effect in ZnO NWs (Supplementary Note 6). More control experiments were conducted to confirm the role played by the pyroelectric effect on the photon-sensing performances of the self-powered $\mathrm{ZPH}$ PDs. Figure 4a presents the short-circuit current response of the PDs under 325-nm illumination (power density of 
$3.7 \times 10^{-3} \mathrm{~W} \mathrm{~cm}^{-2}$ ) in different background environmental temperatures ranging from 27 to $70{ }^{\circ} \mathrm{C}$. The sharp peak of the short-circuit currents related to pyroelectric effect $I_{\text {pyro }}$ photo decreased with increasing the environmental temperature, while the stable plateau of the current controlled by photo-excitation process $I_{\text {photo }}$ remained unchanged. These results further verify the mechanism of light-self-induced pyroelectric effect in $\mathrm{ZnO}$ NWs. Moreover, by removing the perovskite layer, a new configuration of $\mathrm{Si} / \mathrm{ZnO} \mathrm{PD}$ was fabricated and tested as shown in Fig. 4b. Similar $I-t$ characteristics (Fig. 4b) were obtained under $325-\mathrm{nm}$ illumination, confirming the pyroelectric effect was induced within $\mathrm{ZnO}$ instead of other materials.

Pyroelectric effect improved response time of ZPH PDs. Pyroelectric effect enhancements on response time of ZPH PDs were carefully studied by comparing the performances of devices operating under different bias voltages $(0,0.08,0.12$ and 0.3$) \mathrm{V}$ as shown in Fig. 4c-f. Higher applied bias voltage induced larger dark currents, which in turn increased the background temperatures of the device and thus reduced the pyro-potential in $\mathrm{ZnO} \mathrm{NWs}$ as shown as the insets in Fig. $4 \mathrm{c}-\mathrm{f}$. I- $t$ characteristic presented in Fig. 4c-f obviously indicates the gradual disappearance of pyroelectric effect-induced sharp peak as increasing the bias voltage. A double-exponential function ${ }^{10}$ fitting of the $I-t$ curves was performed to calculate the response time of $\mathrm{ZPH}$ PDs under 0 and $0.3 \mathrm{~V}$ bias voltage as summarized in Supplementary Note 7 and Supplementary Fig. 9, with the corresponding fitting parameters shown as the insets. The rising time and the fall time are $53 \mu$ s (Supplementary Fig. 9b) and $63 \mu \mathrm{s}$ (Supplementary Fig. 9c) for the self-powered ZPH PDs under zero bias, respectively. Considering the system response ( $\sim 28 \mu \mathrm{s})$ of the optical chopper switching the light signals, the actual response time of these PDs could be faster. Following the same fitting method, the rising time and fall time of these PDs operating under $0.3 \mathrm{~V}$ bias were calculated as $5.4 \mathrm{~s}$ (Supplementary Fig. 9d) and $8.9 \mathrm{~s}$ (Supplementary Fig. 9e), respectively. Therefore, the photon response time of ZPH PDs was reduced from several seconds to few microseconds (by $10^{5}$ time), by light-self-induced pyroelectric effect in $\mathrm{ZnO} \mathrm{NWs}$. Furthermore, these results also indicate essentiality to configure such PDs in a self-powered manner without applying any bias voltages to maximize the enhancements of pyroelectric effect.

\section{Discussion}

These self-powered ZPH PDs possess novel, unique and complement features in comparison with conventional photosensing devices on the basis of top-down or bottom-up approaches. First, the long-overlooked pyroelectric effect induced by ultraviolet illuminations was employed to enhance the performances of photosensing. This is a fundamentally new mechanism that relies on the naturally existing temperature changes on ultraviolet illuminations. Compared with traditional methods ${ }^{16,17}$ to improve the response time and enhance the general performances of PDs, the pyro-phototronic approach demonstrated in this work is easier, faster and less costly. Moreover, this approach can be extended to other pyroelectric semiconductor-material combinations, including visible and/or infrared-sensitive materials, and thus broaden the spectra ranges for more practical applications. Second, selfpowered PDs operated under zero bias were achieved by forming heterostructures between $\mathrm{ZnO}$ and perovskite. This configuration not only provides energy-efficient ultravioletsensing performances but also maintains low background temperatures by minimizing the dark current $(<0.1 \mathrm{nA})$ to maximize the enhancements of pyroelectric effect. Third, the response time of these self-powered $\mathrm{ZPH}$ PDs was improved through pyroelectric effect from $5.4 \mathrm{~s}$ to $53 \mu \mathrm{s}$ at the rising edge, and $8.9 \mathrm{~s}$ to $63 \mu \mathrm{s}$ at the falling edge by five orders of magnitudes. The specific detectivity $D^{\star}$ and the responsivity $R$ of the PDs were both enhanced by $322 \%$. The newly designed ultrafast ultraviolet PDs operating under zero bias may lead to further explorations in ultrafast optics, nonlinear optics, optothermal detections, computational memories and biocompatible optoelectronic probes.

\section{Methods}

Fabrication process of ZPH PDs. $\mathrm{CH}_{3} \mathrm{NH}_{3} \mathrm{I}$ was synthesized and purified following the previously reported method ${ }^{34}$. The synthesized $\mathrm{CH}_{3} \mathrm{NH}_{3} \mathrm{I}$ powder was mixed with $\mathrm{PbI}_{2}$ (Aldrich) at 1:1 mol ratio in N,N-Dimethylmethanamide (DMF) at $60^{\circ} \mathrm{C}$ for $12 \mathrm{~h}$, followed by filtering twice using $13-\mathrm{mm}$ diameter and 0.45 - $\mathrm{mm}$ pore polyvinylidene difluoride syringe filter (Whatman). The derived product was used as a coating solution for the formation of $\mathrm{MAPbI}_{3}$. Then, $\mathrm{MAPbI}_{3}$ perovskite DMF solution with $40 \mathrm{wt} \%$ was prepared for further usages. Meanwhile, the FTO glasses (Sigma-Aldrich, $13 \Omega^{-2}$ ) were ultrasonically cleaned for $5 \mathrm{~min}$ in acetone, distilled water and ethanol, consecutively. Next, a layer of 50-nm $\mathrm{ZnO}$ seed layer was deposited on FTO glasses by radio frequency magnetron sputtering at room temperature (PVD75 system, Kurt. J. Lesker Company). The coated FTO glasses were then placed into the mixed nutrient solutions $\left(0.02 \mathrm{M} \mathrm{Zn}\left(\mathrm{NO}_{3}\right)_{2}\right.$ and $0.02 \mathrm{M}$ HMTA) for $\mathrm{ZnO}$ NWs growth via a hydrothermal method in a mechanical convection oven (model Yamato DKN400, Santa Clara, CA, USA) at $85^{\circ} \mathrm{C}$ for $30 \mathrm{~min}$. To get separated $\mathrm{ZnO}$ NWs, $0-5 \mathrm{ml}$ ammonium hydroxide (Sigma-Aldrich) was added per $100 \mathrm{ml}$ mixing solution. After cooling down the whole system, the product was washed with ethanol and distilled water, collected and vacuum-dried at $100^{\circ} \mathrm{C}$ for $1 \mathrm{~h}$. A 40 -wt $\%$ perovskite DMF solution was spin-coated on the dried $\mathrm{ZnO}$ array at 4,000 r.p.m., followed by spin-coating the spiro-OMeTAD as a hole transport thin layer with a spin coater (SCS 6800). Another thin layer of $\mathrm{Cu}(250 \mathrm{~nm})$ was deposited as the bottomelectrode afterwards. Testing wires were connected to top and bottom electrodes by silver paste. Finally, a thin layer of kapton tape was employed to fix the testing wires and to improve its resistance to environmental contaminations and corrosions.

Material characterizations. Detailed microscopic structures of $\mathrm{ZnO}$ NWs, perovskite, spiro-OMeTAD and copper film were characterized using scanning electron microscope (Hitachi SU8010), PANalytical X'Pert PRO diffractometer (Almelo, the Netherlands) with $\mathrm{Cu} \mathrm{KR}$ radiation $(\lambda=0.15418 \mathrm{~nm})$ for X-ray diffraction patterns of the $\mathrm{MAPbI}_{3}$ perovskite.

Optical and electrical measurements. Transmission spectra of $\mathrm{ZnO}$ and $\mathrm{ZnO} /$ perovskite were measured by a ultraviolet-visible spectrophotometer (J $\Lambda$ SCO V-630). I-V characteristics of the device were measured and recorded with a self-developed, computer-controlled measurement system with Stanford SRS Low-noise current preamplifier (SR570)/SRS low-noise voltage preamplifier (SR560) in conjunction with a GPIB controller (GPIB-USB-HS, NI 488.2). The optical input stimuli were provide by a $\mathrm{He}-\mathrm{Cd}$ dual-colour laser (wavelength $=325$ and 442 nm, Model No. KI5751I-G, Kimmon Koha Co, Ltd). A continuously variable filter was used to control the light power density, which was measured with a thermopile powermeter (Newport 818 P-001-12). An objective was used to expand 325 and $442 \mathrm{~nm}$ laser to illuminate the whole device. The effective size of the device is $3 \times 3 \mathrm{~mm}$. A hot plate was used to control and change the background environmental temperatures.

\section{References}

1. Wang, X. D., Song, J. H., Liu, J. \& Wang, Z. L. Direct-current nanogenerator driven by ultrasonic waves. Science 316, 102-105 (2007).

2. Huang, M. H. et al. Room-temperature ultraviolet nanowire nanolasers. Science 292, 1897-1899 (2001)

3. Pan, C. F. et al. High-resolution electroluminescent imaging of pressure distribution using a piezoelectric nanowire LED array. Nat. Photonic 7, 752-758 (2013).

4. Wu, W. Z., Wen, X. N. \& Wang, Z. L. Taxel-addressable matrix of verticalnanowire piezotronic transistors for active and adaptive tactile imaging. Science 340, 952-957 (2013).

5. Yu, R. et al. Piezo-phototronic Boolean logic and computation using photon and strain dual-gated nanowire transistors. Adv. Mater. 27, 940-947 (2015).

6. Park, W. I., Kim, J. S., Yi, G. C. \& Lee, H. J. ZnO nanorod logic circuits. Adv. Mater. 17, 1393-1397 (2005)

7. Monroy, E., Omnes, F. \& Calle, F. Wide-bandgap semiconductor ultraviolet photodetectors. Semicond. Sci. Technol. 18, R33-R51 (2003).

8. Hatch, S. M., Briscoe, J. \& Dunn, S. A self-powered ZnO-nanorod/CuSCN UV photodetector exhibiting rapid response. Adv. Mater. 25, 867-871 (2013).

9. Kim, D. C., Jung, B. O., Kwon, Y. H. \& Cho, H. K. Highly sensible ZnO nanowire ultraviolet photodetectors based on mechanical schottky contact. J. Electrochem. Soc. 159, K10-K14 (2012) 
10. Soci, C. et al. ZnO nanowire UV photodetectors with high internal gain. Nano Lett. 7, 1003-1009 (2007).

11. Wang, Z. et al. Optimizing performance of silicon-based pn junction photodetector by piezo-phototronic effect. ACS Nano 8, 12866-12873 (2014).

12. Kind, H., Yan, H. Q., Messer, B., Law, M. \& Yang, P. D. Nanowire ultraviolet photodetectors and optical switches. Adv. Mater. 14, 158-160 (2002).

13. Ahn, S. E. et al. Photoresponse of sol-gel-synthesized $\mathrm{ZnO}$ nanorods. Appl. Phys. Lett. 84, 5022-5024 (2004).

14. Keem, K. et al. Photocurrent in $\mathrm{ZnO}$ nanowires grown from $\mathrm{Au}$ electrodes. Appl. Phys. Lett. 84, 4376-4378 (2004).

15. Jeong, M. C., Oh, B. Y., Lee, W. \& Myoung, J. M. Optoelectronic properties of three-dimensional ZnO hybrid structure. Appl. Phys. Lett. 86, 103105 (2005).

16. Hu, Y. F. et al. Supersensitive fast-response nanowire sensors by using schottky contacts. Adv. Mater. 22, 3327-3332 (2010).

17. Cheng, G. et al. $\mathrm{ZnO}$ nanowire Schottky barrier ultraviolet photodetector with high sensitivity and fast recovery speed. Appl. Phys. Lett. 99, 203105 (2011).

18. Heiland, G. \& Ibach, H. Pyroelectricity of zinc oxide. Solid State Commun. 4, 353-356 (1966)

19. Hsiao, C. C. \& Yu, S. Y. Improved response of $\mathrm{ZnO}$ films for pyroelectric devices. Sensors 12, 17007-17022 (2012).

20. Hsiao, C. C., Huang, S. W. \& Chang, R. C. Temperature field analysis for $\mathrm{ZnO}$ thin-film pyroelectric devices with partially covered electrode. Sensor Mater. 24, 421-441 (2012).

21. Bie, Y. Q. et al. Self-powered, ultrafast, visible-blind UV detection and optical logical operation based on $\mathrm{ZnO} / \mathrm{GaN}$ nanoscale p-n junctions. Adv. Mater. 23, 649-653 (2011)

22. Xu, S. et al. Self-powered nanowire devices. Nat. Nanotechnol. 5, 366-373 (2010).

23. Lee, Y. et al. High-performance perovskite-graphene hybrid photodetector. Adv. Mater. 27, 41-46 (2015).

24. Mei, A. Y. et al. A hole-conductor-free, fully printable mesoscopic perovskite solar cell with high stability. Science 345, 295-298 (2014).

25. Malinkiewicz, O. et al. Perovskite solar cells employing organic chargetransport layers. Nat. Photonics 8, 128-132 (2014).

26. Li, D., Dong, G. F., Li, W. Z. \& Wang, L. D. High performance organicinorganic perovskite-optocoupler based on low-voltage and fast response perovskite compound photodetector. Sci. Rep. 5, 7902 (2015).

27. Dou, L. T. et al. Solution-processed hybrid perovskite photodetectors with high detectivity. Nat. Commun. 5, 5404 (2014).

28. Hu, X. et al. High-performance flexible broadband photodetector based on organolead halide perovskite. Adv. Funct. Mater. 24, 7373-7380 (2014).

29. Xia, H. R., Li, J., Sun, W. T. \& Peng, L. M. Organohalide lead perovskite based photodetectors with much enhanced performance. Chem. Commun. 50, 13695-13697 (2014)

30. Ku, Z. L., Rong, Y. G., Xu, M., Liu, T. F. \& Han, H. W. Full printable processed mesoscopic $\mathrm{CH} 3 \mathrm{NH} 3 \mathrm{PbI} 3 / \mathrm{TiO} 2$ heterojunction solar cells with carbon counter electrode. Sci. Rep. 3, 3132 (2013).
31. Kim, H. S. et al. Lead iodide perovskite sensitized all-solid-state submicron thin film mesoscopic solar cell with efficiency exceeding 9\%. Sci. Rep. 2, 591 (2012).

32. Liu, X. et al. All-printable band-edge modulated $\mathrm{ZnO}$ nanowire photodetectors with ultra-high detectivity. Nat. Commun. 5, 4007 (2014).

33. Konstantatos, G. \& Sargent, E. H. Nanostructured materials for photon detection. Nat. Nanotechnol. 5, 391-400 (2010).

34. Im, J. H., Lee, C. R., Lee, J. W., Park, S. W. \& Park, N. G. 6.5\% efficient perovskite quantum-dot-sensitized solar cell. Nanoscale 3, 4088-4093 (2011).

\section{Acknowledgements}

This research was supported by the US Department of Energy, Office of Basic Energy Sciences (Award DE-FG02-07ER46394), the National Science Foundation (DMR-1505319), National Natural Science Foundation of China (Grant Nos. 51432005, 11104016 and 11574033) and the 'thousands talents' programme for pioneer researcher and his innovation team. We thank Professor M. El-Sayed, Dr P. Szymanski and D. O’Neil for discussions.

\section{Author contributions}

Z.L.W., Z.W. and R.Y. conceived the idea. Z.W. and R.Y. designed the experiments. Z.W. and R.Y. performed the experiments. Z.W., R.Y. and C.P. analysed the data. Z.W., R.Y. and Z.L. characterized the materials. R.Y., Z.W. and Z.W. wrote the paper. All authors discussed the results and commented on the manuscript. Z.W. and R.Y. contributed equally to this work.

\section{Additional information}

Supplementary Information accompanies this paper at http://www.nature.com/ naturecommunications

Competing financial interests: The authors declare no competing financial interests.

Reprints and permission information is available online at http://npg.nature.com/ reprintsandpermissions/

How to cite this article: Wang, Z. et al. Light-induced pyroelectric effect as an effective approach for ultrafast ultraviolet nanosensing. Nat. Commun. 6:8401 doi: 10.1038/ncomms9401 (2015)

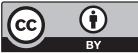

This work is licensed under a Creative Commons Attribution 4.0 International License. The images or other third party material in this article are included in the article's Creative Commons license, unless indicated otherwise in the credit line; if the material is not included under the Creative Commons license, users will need to obtain permission from the license holder to reproduce the material. To view a copy of this license, visit http://creativecommons.org/licenses/by/4.0/ 